\title{
Barriers to living kidney donation among relatives of patients with chronic renal failure in south Africa
}

\author{
Pretty Mbeje $^{1}$, Mary Moleki ${ }^{2}$, Makombo Ganga-Limando ${ }^{3 *}$ \\ ${ }^{1-3}$ Department of Health Studies University of South Africa (UNISA) \\ ${ }^{3}$ Department of Health Studies University of South Africa (UNISA) \\ *Corresponding author E-mail: gangam@unisa.ac.za
}

\begin{abstract}
Background: The number of patients with chronic renal failure awaiting kidney transplantation has been increasing in the country. There is a great awareness regarding the importance of kidney donation among family members and the public at large. But little is known about their reluctance to donate kidney.

Aim: The aim of this study was to explore and describe barriers to living kidney donation among relatives of patients with chronic renal failure undergoing haemodialysis in one of the provinces in South Africa.

Methods: The study used qualitative, descriptive, exploratory and contextual design. Purposive sampling was carried out to select close family members and relatives of patients undergoing haemodialysis. Data was collected through individual face to face in-depth interviews with 25 participants. Interviews were recorded and analysed using thematic content analysis approach.

Results: This study identified six major barriers to living kidney donation among relatives of patients with chronic renal failure undergoing haemodialysis. They included: cultural beliefs, mistrust, fear, lack of information, being old, and pre-existing medical conditions.

These barriers are well documented in the literature.

Conclusion: The study has direct implication for the quality of life of patients with chronic renal failure. Kidney transplantation is associated with drastic improvements in survival and quality of life as well as considerable cost savings, compared with haemodialysis or peritoneal dialysis. It cannot be achieved without organ donors. Therefore, extensive public education aimed at ad-dressing factors restraining people to donate organ is needed.
\end{abstract}

Keywords: Barriers to Kidney Donation, Chronic Renal Failure, Haemodialysis, Patients' Relatives, South Africa.

\section{Introduction}

Chronic renal failure is increasingly being recognised as a global health problem. Chronic renal failure, also known as chronic kidney disease is a progressive, irreversible renal function in which the body fails to maintain metabolic, fluid and electrolyte balance, resulting in uraemia or azotaemia (Smeltzer et al. 2008). It causes an inexorable attrition of nephron function leading to end-stage renal disease or end-stage renal failure. In turn, end-stage renal failure represents a clinical state in which there has been an irreversible loss of endogenous renal function (Erdie et al. 2010).

Authors (White et al. 2008) argued that the recognition of chronic kidney disease as a public health problem has evolved, in part, from the acceptance of the conceptual model, definition, and classification of chronic kidney disease or chronic renal failure proposed by the National Kidney Foundation and the Kidney Disease Outcome Quality Initiative in 2002. However, the lack of national registries and representative surveys, make it difficult to estimating the burden of end-stage renal disease in low and middle income countries.

A growing body of literature has demonstrated that although renal replacement therapy for patients with chronic renal failure is cost effective, this disease continues to impact on health-related quality of life. White et al (2008, p229) maintain that "the global burden of end-stage renal failure is concealed behind the statistics which reflect only the number of people treated, not those who die of kidney failure. The number of patients with chronic renal failure awaiting kidney transplantation is drastically increasing and living donor kidney transplantation is the treatment of choice and has economic and quality-of-life advantages. The success of kidney transplants depends on a constant supply of suitable donors. Insufficient organ donors results in the increase of the number of patients undergoing haemodialysis and saturation of haemodialysis units. Saturation of the haemodialysis units can only be curbed if relatives come forward for live-related transplants.

It is known that organ donation is influenced by several factors. Insufficient organ donor and transplant programs, the structural issues within the health systems, and the cost of pharmaceuticals are identified as barriers to the delivery of efficient and costeffective renal replacement therapy (Yang 2007). In addition, the traditional belief about the body having to remain intact to enter the spirit world has been cited as a barrier to the concept of kidney donation (Andrews 2008). These issues cannot be ignored by health professionals. The understanding of these barriers may assist the health professionals in designing interventions aimed at strengthening the organ and transplant programs, thereby increasing the pool of organ donors.

This study focused on the relatives of patients with chronic renal failure who were undergoing haemodialysis in the province of KwaZulu-Natal in South Africa. The province has only three provincial hospitals that offer haemodialysis to patients with chronic 
renal failure that are awaiting renal transplantation. The aim of the study was to explore and describe barriers to kidney donation among relatives of patients with chronic renal failure undergoing haemodialysis in one of the provinces in South Africa.

\section{Methods}

\subsection{Design}

A qualitative descriptive design was used to explore and describe barriers to kidney donation among relatives of patients suffering from chronic renal failure. A qualitative design allows the researchers to uncover the individuals' subjective perceptions and interpretations of a phenomenon in a real life situation (Polit \& Beck 2012). In this study the researchers were interested in understanding barriers to kidney donation as perceived by people who are closely related to the patients waiting for kidney donation.

\subsection{Setting}

The study was conducted in one of the provinces (KwaZulu-Natal) of South Africa. They were only three hospitals with haemodialysis units in province during the study period. All three were included in the study.

\subsection{Population and sampling}

The population of this study consisted of the relatives of patients suffering from chronic renal failure that were waiting for kidney donation and undergoing haemodialysis in three public hospitals. Purposive sampling was used to select the relatives of the above patients who met the following inclusion criteria: (1) be a near relative to the patients and having accompanied the patients to the haemodialysis unit at least once in the past three months; (2) be more than 18 year-old and mentally fits; and (3) be able to speak English and willing to participate in the study. Forty-five relatives met the above criteria and were considered for interviews. They were identified by the researchers while accompanying the patients at the haemodialysis units.

\subsection{Data collection}

The researchers used semi-structured individual interviews to collect data between November 2011 and March 2012. The interview schedule included one main open-ended question "what do you consider as barriers to donate kidney to your sick relative as prescribed by the doctor?" Probing questions were used when appropriate to enhance the richness of data. All the participants agreed to be interviewed at their residences. Each interview lasted between 40 to 45 minutes. The researchers used field notes to capture body language and facial expression of the interviewees. Data collection ceased after 25 interviews when saturation of themes was reached.

\subsection{Data analysis}

The interviews were digitally recorded; checked for quality, and immediately transcribed verbatim in order to refrain from missing relevant data. A follow-up was held to review and verify the transcripts with the informants the following day. The researchers used thematic content analysis to process the transcribed data (Creswell 2013).The emerged theme and sub-themes were organized and interpreted to draw relationships between codes to aid easy presentation.

\subsection{Ethical considerations}

The study received ethical clearance from the Ethics Committees of the Department of Health Studies, University of South Africa, and the National Department of Health. The researchers adhered to all ethical principles related to research involving human subjects. Participants were informed about the study, their rights to free participation, confidentiality, privacy, and to withdraw from the study at any time. Written and signed informed consent was obtained from the participant before the interviews. Data collected were treated with confidentiality and anonymity throughout the management and analysis processes.

\subsection{Scientific rigour}

The researchers followed the principles outlined by Polit and Beck to ensure trustworthiness of the study (Bryman 2012). These principles include: confirmability, dependability, neutrality, and transferability. The researchers achieved confirmability through prolonged engagement and triangulation of data, using independent coding. Dependability was achieved through the use of independent coder at different phases of the coding process. Neutrality was ensured by keeping appropriate distance between the researchers and the participants. Transferability was observed by providing detailed descriptions of the participants' description of the phenomenon, as well as the researcher's observations in reporting the findings.

\section{Results}

\subsection{Characteristics of the participants}

The participants' ages ranged from 25 to 65 year-old. Nineteen were females and six were males. Of the 25 participants, 20 $(80.0 \%)$ were illiterates, and $5(20.0 \%)$ had undergraduate qualifications; $20(80.0 \%)$ were Blacks, and $5(20.0 \%)$ were Indians; 15 (60.0\%) have been accompanying the patients to the haemodialysis units for at least 18 months; $4(16.0 \%)$ have been accompanying them for 12 months; and $6(24.0 \%)$ for a period of 6 months.

\subsection{Themes}

Barriers to living kidney donation among the relatives of patients with chronic renal failure undergoing haemodialysis were classified into two main themes: psychosocial related barriers, and individual and biomedical related barriers. Six sub-themes emerged from the participants' descriptions of what they considered as barriers to donate kidney. Table 1 provides a summary of the themes and sub-themes that emerged from data with the number of times each sub-theme was mentioned. Excerpts from participants' narratives are used to support the emerged sub-themes.

Table 1: Themes with Related Sub-Themes

\begin{tabular}{lll}
\hline \multirow{2}{*}{ Themes } & Sub-themes & $\begin{array}{l}\text { Number of } \\
\text { times } \\
\text { mentioned }\end{array}$ \\
\hline \multirow{3}{*}{ Psychosocial related barriers } & Cultural beliefs & 14 \\
& Mistrust of partner & 9 \\
& Fear & 18 \\
& Lack of information & 19 \\
Individual and biomedical & Being old & 5 \\
related barriers & Pre-existing medical & 22 \\
\hline
\end{tabular}

\subsubsection{Psychosocial related barriers}

As indicated in Table 1, cultural beliefs, mistrust, fear, and lack of information are the three sub-themes related to psychosocial barriers to living kidney donation among the participants.

\section{Cultural belief}

This sub-theme was mentioned by 14 participants as a barrier to kidney donation. It emerged from their descriptions that kidney donation or the donation of any organ for that matter was against the cultural. They believed that the ancestors will reject them if they were buried without the kidney. 
My ancestors will not accept me in my family when I die because I would not be the "whole" if I donate my kidney.

\section{Mistrust}

This sub-theme was mentioned by 9 participants who were all married women. They felt unwise to donate kidney to their husbands as they may leave them at any time.

I can only donate to my parents and my kids but not my husband as he can divorce me and take another woman forgetting that he's surviving because of my kidney transplanted to him.

\section{Fear}

This sub-theme emerged from the descriptions of 18 participants. Fear was associated with the medical process and individual future needs.

For some, donating organ to a relative was a sensitive issue which they felt difficult to discuss.

It is a very sensitive issue which is even difficult to start discussing and I feel sorry for my husband. I'm personally very scared of donating.

Others were afraid to donate due to uncertainty about their own needs in the future. They felt that it was very risky to live with one kidney.

What if I also become ill or what if my one and only son become ill and also needs a kidney? I better wait for another person to donate.

\section{Lack of information}

This sub-theme emerged from the descriptions of 19 participants They associated kidney donation with negative health consequences.

Some participants felt that they will not be able to bear children after kidney donation. It was expressed with feelings of anxiety.

I don't want to donate kidney because it will not be possible for me to bear children after donation.

Others were not keen to donate because of lack of knowledge regarding the recovery process.

I am not looking forward to donate as I am not sure how long it will take me to recover after kidney donation.

Lack of information was also associated with the belief that kidney donation was not needed if the patient is doing well under the haemodialysis treatment.

Kidney donation is a good thing but I've never taken it serious since my brother is doing well on haemodialysis.

\subsubsection{Individual and biomedical related barriers}

As indicated in Table 1, being old, and pre-existing medical conditions emerged as individual related barriers to living kidney donation among the participants.

\section{Being old}

Being old as a barrier was mentioned by 5 participants who were more than 50 year-old. It was associated with chronic medical conditions.

I'm too old to donate and worse diabetic, so if anyone who is a guardian angel can donate, that will really be appreciated.

\section{Pre-existing medical conditions}

This sub-theme was mentioned by 22 participants who were willing and intended to donate kidney but they failed the screening tests due to poor health status. These participants commenced the renal transplant workup programme, but they were disqualified after the screening tests.

Both my daughters were interested to donate for their only brother; one was excluded at the early stages of the investigations because of the heart problem that was identified. With the younger daughter, it was detected that the blood supply to her own kidney is not good and is currently being investigated for that.

\section{Discussion}

This study identified six major barriers to living kidney donation among relatives of patients with chronic renal failure undergoing haemodialysis. The decision to donate kidney to a relative in need is negatively affected by psychosocial factors (cultural beliefs, mistrust, fear, and lack of information) and biomedical factors (old-age and poor health status). These barriers are well documented in the literature.

The link between the psychosocial factors identified as barriers in this study has been established by previous studies (Mathew et al. 2005, Oliver et al. 2011). In a study of family issues implicit in living donation, it was revealed that despite the willingness of family members to donate organs to their relatives, the decision to donate organ was greatly influenced by cultural beliefs and the complexity of relationships within the family (Crombie \& Franklin 2006). Differences exist on the role of the lack of information on the process of kidney donation as a barrier. Some studies identified lack of information on the process of kidney donation as a barrier (Davis \& Randhawa 2004, Waterman et al. 2006), while others did not show any link between the level of knowledge or information and the decision to donate organ (Powe \& Boulware 2002, Barcellos et al. 2005).

The results of this study suggest that the issue of living kidney donation is highly emotional as expressed with the feelings of mistrust and fear. Previous studies attributed those feelings as barriers to living kidney donation among Blacks and Hispanic Americans (Alvaro et al. 2008, Wing et al. 2010).

The results on psychosocial barriers highlight the need for public education regarding the importance of kidney donation for patients with chronic renal failure. Many relatives are willingness to donate kidney but this willingness is negatively affected by their cultural beliefs, lack of information, feelings of mistrust and fear. These factors can be changed through well-structured public education. It is argued that well-structured public education on kidney donation will provide the family or relatives of the patients with chronic renal failure time to work though their reactions and feelings about kidney donation at an earlier stage of their relatives' condition, before the situation becomes critical, so that they can deal with these negative perceptions.

Age and pre-existing medical conditions are commonly identified as barriers to living kidney donation during donor identification by health professionals (Boulware et al. 2002, Young \& Gaston 2002). This is a challenge to health professionals in that as much as they emphasize the importance of kidney donation; they have to deal with respect the individual's religious beliefs and cultural values.

\section{Conclusion}

The study has direct implication for the quality of life of patients with chronic renal failure. Kidney transplantation is associated with drastic improvements in survival and quality of life as well as considerable cost savings, compared with haemodialysis or peritoneal dialysis. Kidney transplant results in greater ability of patients to participate productively in the community. However, thus cannot be achieved without organ donors. Therefore, extensive public education aimed at addressing factors restraining people to donate organ is needed.

\section{Acknowledgement}

We wish to thank all the relatives who participated who participated in the study and the staff of the haemodialysis units for their support.

\section{References}

[1] Alvaro EM, Siegel JT, Truscott D, Lisa N, Crane WD \& Dominick A (2008) Living kidney donation among Hispanics; a qualitative examination of barriers and opportunities. Prog Transplant 18(4), 243-250. [PubMed]

[2] Andrews PA (2008) early identification of chronic kidney disease in adults in primary and secondary care: A commentary on NICE guide- 
line No 73. British Journal of Diabetes and Vascular Disease 8(6), 257-262. [PubMed]

[3] Barcellos FC, Araujo CL \& da Costa JD (2005) Organ donation; population based study. Clinical Transplant 19, 33-37. [PubMed]

[4] Boulware LE, Ratner LE, Sosa JA, and Cooper LA, LaViest TA \& Powe NR (2002) Determinants of willingness to donate living related and cadaveric organs: identifying opportunities for intervention. Transplantation 73, 1683-1691. [PubMed]

[5] Bryman A (2012) Social Research Methods, $4^{\text {th }}$ edition, Oxford University Press.

[6] Creswell JW (2013) Qualitative Inquiry \& Research Design: Choosing Among Five Approaches. $3^{\text {rd }}$ edition, Sage- London.

[7] Crombie AK \& Franklin PM (2006) Family Issues Implicit in Living Donation. Mortality 11(2), 196-210.

[8] Davis C \& Randhawa G (2004) "Don't know enough about it": awareness and attitudes toward organ donation and transplantation among the black Caribbean and black African population in Lambeth, Southwark, and Lewisham, United Kingdom. Transplantation 78, 420425.

[9] Erdei V, Duret N \& Mironov G (2010) Legal issues related to donation of organs, tissues and cells of human. EIRCP Proceedings 5,133137.

[10]Mathew T, Faull R \& Snelling PL (2005) the shortage of kidney for transplantation in Australia. Medical Journal Australia 182, 204-205

[11]Oliver M, Woywodt A, Ahmed A \& Saif I (2011) Organ donation, transplantation and religion. Nephrology Dialysis Transplantation 26(2), 1745.

[12]Polit FD \& Beck CT (2012) Nursing Research: generating and assessing evidence for nursing practice. $9^{\text {th }}$ edition, Philadelphia: Lippincott Williams and Wilkins.

[13]Powe NR \& Boulware LE (2002) the uneven distribution of kidney transplants: Getting at the root causes and improving care. American Journal Kidney Diseases 40(4), 861-863. [PubMed]

[14]Smeltzer SC, Bare BG, Hinkle JL \& Cheever KH (2008) Brunner and Suddarth textbook of medical and surgical nursing, $11^{\text {th }}$ edition, Lippincott: Philadelphia.

[15] Waterman AD, Stanley SL, Covelli T, Hazel E, Hong BA, Brennan DC (2006) living donation decision-making: recipients' concerns and education needs. Prog Transplant 16, 17-23. [PubMed]

[16] White SL, Chadban SJ, Jan S, Chapman JR \& Cass A (2008) How can we achieve global equity in provision of renal replacement therapy? Bulletin of the World Health Organization 86(3), 229-240.

[17] Yang RC (2007). Insurability of living donors: a systematic review. American Journal of Transplantation 7(6), 1542-1551.

[18]Young CJ \& Gaston RS (2002) African Americans and renal transplantation: Disproportionate need, limited access, and impaired outcomes. Is J Med Sc 323, 94-96. [PubMed]

[19]Wing FL, Reese PP, Mulgaonkar S \& Patel AM (2010) Barriers to living donor kidney transplantation among black or older transplant candidates. Clinical Journal of American Society of Nephrology 5(12), 2338-2347. [PubMed] 